\title{
Optimal Prestress Investigation on Tensegrity Structures Using Artificial Fish Swarm Algorithm
}

\author{
Xiaodong Feng $\mathbb{D D}^{1,2,3}$ Wanpeng Zhang, ${ }^{1}$ Yaozhi Luo, ${ }^{2}$ and Sergio Zlotnik ${ }^{4}$ \\ ${ }^{1}$ College of Civil Engineering, Shaoxing University, Shaoxing 312000, China \\ ${ }^{2}$ College of Civil Engineering and Architecture, Zhejiang University, Hangzhou 310058, China \\ ${ }^{3}$ Technology Center, Zhejiang Jinggong Steel Building Co., Ltd., Shaoxing 312000, China \\ ${ }^{4}$ Laboratori de Càlcul Numèric (LaCàN), E.T.S. Ingenieros de Caminos, Universitat Politècnica de Catalunya, Barcelona Tech, \\ Barcelona 08034, Spain
}

Correspondence should be addressed to Xiaodong Feng; xiaodong.feng@csu.edu.cn

Received 9 February 2020; Revised 26 May 2020; Accepted 27 August 2020; Published 8 September 2020

Academic Editor: Daniele Baraldi

Copyright $\odot 2020$ Xiaodong Feng et al. This is an open access article distributed under the Creative Commons Attribution License, which permits unrestricted use, distribution, and reproduction in any medium, provided the original work is properly cited.

To obtain the optimal uniform prestress of a tensegrity structure with geometric configuration given, a novel method is developed for prestress design of tensegrity structures by utilizing the artificial fish swarm algorithm (AFSA). In the beginning, the formfinding process is implemented by solving a linear homogeneous system concerning the self-equilibrium system. The issue is subsequently performed as a minimum problem by regulating the value of an objective function where the unilateral condition and the stress uniformity condition are entirely considered. The AFSA is adopted to search for the global minimum, leading to a set of initial prestresses that guarantee all the above conditions. Two illustrative examples have been fully studied to prove the accuracy and efficiency of the presented approach in prestress design of tensegrities according to the practical requirements. Furthermore, the numerical examples investigated in this paper confirm that the AFSA has explicit advantages of rapid convergence and overcoming the local minima.

\section{Introduction}

Tensegrity structures are self-stressed lightweight structures which consist of two different components, known as continuous cables in tension and interval struts in compression. Normally, regardless of external forces, the structure could maintain stability due to the contribution of self-stressed cables. It is acknowledged that lightweight and tunable stiffness are the two basic characteristics of tensegrity structures due to the fact that prestress stiffens the structure and all components are axially loaded. Accordingly, since the concept of "tensegrity" arose in the early 1950 s, it has received vast interest among engineers and scientists throughout domains such as aerospace [1], biology [2-4], mathematics [5], architecture [6], robotics [7-9], and civil engineering [10-14] to materials [15].

Before feasible initial prestresses are assigned, a certain shape would not be formulated due to the fact that the rigidity of a tensegrity is determined by its self-stressed equilibrium concerning struts and cables. As is known to all, in the process of prestress design of tensegrity structures, it is usually difficult to acquire the self-balancing configuration satisfying specific features appointed by researchers. Hence, to effectively and rationally explore the relationship between the distribution of appropriate prestress and the initial geometry configuration becomes the core issues in designing tensegrities. Under normal circumstances, after the determination of initial structural shape, the morphological analysis of a tensegrity structure turns into searching for a feasible distribution of initial prestress, known as the formfinding or force-finding. To design and optimize the shape of tensegrities, a vast number of methods have been proposed over the past 40 decades [16-25]. Recently, the authors of [26] studied the self-equilibrium and geometrical stability properties of tensegrities by utilizing the genetic algorithm. Following this trace, the authors of [27] raised the role of 
optimal prestress design which is suitable for cable domes. The study in [28] raised a prestress design method of tensegrity structures via analyzing the structural stiffness matrix. The study in [29] developed a new form-finding procedure for truncated polyhedral tensegrity structures by integrating the classical force density method into the genetic algorithm. The study in [30] formulated an analytical form-finding method of tensegrities based on Faddeev-LeVerrier algorithm. The study in [31] investigated the optimal prestress design of tensegrity grid structures by introducing the Interior-Point Method into the formfinding process. The study in [14] determined the feasible prestress modes for cable-strut structures on the basis of particle swarm algorithm.

Although there have been plenty of approaches formulated for prestress design of tensegrities, the vast majority of available algorithms can only deal with cases that own single self-stress state mode; however, they may not be efficient enough in solving problems with multiple states of self-stress modes, where the structural force densities of all components can be depicted as a linear combination of these mutually independent self-stress modes. Generally, it is not easy to acquire the solution (a feasible vectorial base) from the calculation of the abovementioned self-stress modes. On the other hand, the initial force densities of members are expected to be uniform as much as possible to make full use of material. In other words, minimization of dispersion between elemental force densities of the specified groups also plays an important role in an actual project. Consequently, the determination of distribution of optimal feasible initial prestress and the corresponding intensity is indeed the core step in the initial design of a tensegrity. And the structural mechanic properties are subsequently investigated as the next step once the intensity and distribution of the initial prestress have been determined.

The artificial fish swarm algorithm, proposed in [32], is a relatively novel swarm intelligent evolutionary computation technique that was inspired by natural feeding behaviors of fish. There are three typical feeding behaviors, known as foraging, swarming, and following. The first one is hunting for food, the second simulates the response to possible danger, and the last one is imitated to raise the chance of obtaining an expected solution, leading to a powerful ability to achieve a global optimization instead of obtaining local minimums. In the past few years, the AFSA has received quite much praise and attention due to its abilities in performing beautiful formulations and converges rapidly to a solution. Hence, it has been widely applied in regions of image and signal processing [33], automatic control [34], communication [35], and geotechnical engineering [36].

Inspired by the above observations, a novel approach is formulated for optimal prestress design of tensegrity structures via AFSA. In the beginning, the procedure is implemented by settling a linear homogeneous problem concerning a self-equilibrium system. The issue is subsequently performed as a minimum problem by regulating the value of an objective function where the unilateral condition and the stress uniformity condition are entirely considered.
Accordingly, the AFSA is adopted to search for the global minimum solution that guarantees all the abovementioned conditions. Finally, two illustrative examples, including both planar and spatial tensegrities, have been investigated comprehensively in prestress design of tensegrity structures according to the requirements of a practical project. The numerical examples presented confirm that AFSA has advantages of overcoming the local minima and rapid convergence.

\section{Basic Principle of the Form- Finding Procedure}

The topology of a $d$-dimensional ( $d=2$ or 3 ) tensegrity can be depicted by the connectivity matrix $\mathbf{C}_{S} \in \mathbf{R}^{b \times n}$, where $n$ and $b$ represent the number of free nodes and structural members, respectively. The $i$ th and $j$ th elements of the $k^{\text {th }}$ row of $\mathbf{C}_{S}$ are set to 1 and-1, respectively:

$$
\mathbf{C}_{S(k, p)}= \begin{cases}1, & \text { for } p=i, \\ -1, & \text { for } p=j, \\ 0, & \text { otherwise, }\end{cases}
$$

where nodes $i$ and $j(i<j)$ are supposed to be connected by member $k$.

Let $\mathbf{x}, \mathbf{y}, \mathbf{z}\left(\in \mathbf{R}^{n}\right)$ represent the nodal coordinate vectors in $x_{-}, y_{-}$, and $z$-directions of the free nodes.

The force density matrix $\mathbf{Q} \in \mathbf{R}^{b \times b}$ is described as

$$
\mathbf{Q}=\operatorname{diag}(\mathbf{q}) \text {, }
$$

where the self-stress coefficients vector is denoted by $\mathbf{q}=\left\{q_{1}, q_{2}, \ldots, q_{b}\right\}^{T} \in \mathbf{R}^{b}$, where each component represents the so-called self-stress coefficient, defined as the force $f_{k}$ to length $l_{k}$ ratio $q_{k}=f_{k} / l_{k}(k=1,2, \ldots, b)$.

As is known to all, the equilibrium equations of a general pin-jointed structure can be written as

$$
\begin{aligned}
& \mathbf{C}^{T} \mathbf{Q C x}=\mathbf{p}_{x}, \\
& \mathbf{C}^{T} \mathbf{Q C y}=\mathbf{p}_{y}, \\
& \mathbf{C}^{T} \mathbf{Q C z}=\mathbf{p}_{z},
\end{aligned}
$$

in which $\mathbf{p}_{x}, \mathbf{p}_{y}, \mathbf{p}_{z}\left(\in \mathbf{R}^{n}\right)$ denote the external loading vectors imposing on structural nodes.

Matric $\mathbf{E} \in \mathbf{R}^{n \times n}$ is then formulated as

$$
\mathbf{E}=\mathbf{C}^{T} \mathbf{Q} \mathbf{C}
$$

noting $\mathbf{E}$ will also be constant with the given force density matrix.

With the ignorance of external loading (including selfweight), no fixed node is required in a tensegrity structure. In such situation, one can describe its shape by relative positions of nodes. Hence, the system can be formulated as a free-form rigid-body structure:

$$
\mathbf{E}\left[\begin{array}{lll}
\mathbf{x} & \mathbf{y} & \mathbf{z}
\end{array}\right]=\mathbf{C}^{T} \mathbf{Q C}\left[\begin{array}{lll}
\mathbf{x} & \mathbf{y} & \mathbf{z}
\end{array}\right]=\left[\begin{array}{lll}
0 & 0 & 0
\end{array}\right] .
$$

The self-equilibrium matrix can then be described as 


$$
\mathbf{A q}=\left(\begin{array}{c}
\mathbf{C}^{T} \operatorname{diag}(\mathbf{C x}) \\
\mathbf{C}^{T} \operatorname{diag}(\mathbf{C y}) \\
\mathbf{C}^{T} \operatorname{diag}(\mathbf{C z})
\end{array}\right) \mathbf{q}=0,
$$

where $\mathbf{A}$ is the so-called structural self-equilibrium matrix. The singular value decomposition is subsequently performed [37]

$$
\mathbf{A}=\mathbf{U V W}^{T},
$$

where $\mathbf{U} \in \mathbf{R}^{d n \times d n}$ and $\mathbf{W} \in \mathbf{R}^{b \times b}$ represent the orthogonal matrices that are described as

$$
\begin{aligned}
\mathbf{U} & =\left[\begin{array}{llll}
\mathbf{u}_{1} & \mathbf{u}_{2} & \cdots & \mathbf{u}_{d n}
\end{array}\right], \\
\mathbf{W} & =\left[\begin{array}{llll}
\mathbf{w}_{1} & \mathbf{w}_{2} & \cdots & \mathbf{w}_{b}
\end{array}\right] .
\end{aligned}
$$

The singular values are arranged in decreasing order as

$$
\mu_{1} \geq \mu_{2} \geq \cdots \geq \mu_{b} \geq 0 .
$$

It is well acknowledged that the bases of vector spaces regarding mechanism and force density of a given tensegrity structure can be achieved from the null space of $\mathbf{A}$ [38]. In other words, the bases of vector spaces of self-stress and mechanism of the structure can be calculated from the null space of $\mathbf{A}$. Accordingly, matrices $\mathbf{U}$ and $\mathbf{W}$ are rewritten as

$$
\begin{aligned}
& \mathbf{U}=\left[\begin{array}{llll|lll}
\mathbf{u}_{1} & \mathbf{u}_{2} & \cdots & \mathbf{u}_{r_{A}} & \mathbf{m}_{1} & \cdots & \mathbf{m}_{\mathrm{dn}-r_{A}}
\end{array}\right],
\end{aligned}
$$

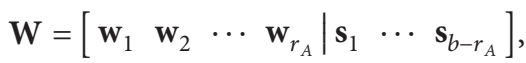

where $r_{\mathbf{A}}=\operatorname{rank}(\mathbf{A}), \mathbf{m} \in \mathbf{R}^{d n-r_{A}}$ represents the $\mathbf{m}(=d n-$ $r_{A}$ ) infinitesimal mechanisms while $\mathbf{s} \in \mathbf{R}^{b-r_{\mathbf{A}}}$ denotes the $\mathbf{s}\left(=d n-r_{A}\right)$ independent self-stress modes. And the mechanisms matrix $\mathbf{M} \in \mathbf{R}^{d n \times\left(d n-r_{A}\right)}$ is defined as

$$
\mathbf{M}=\left[\begin{array}{llll}
\mathbf{m}_{1} & \mathbf{m}_{2} & \cdots & \mathbf{m}_{d n-r_{A}}
\end{array}\right] .
$$

For a complicated tensegrity, normally, $\mathbf{s}$ is larger than one. Hence, the integral feasible self-stress mode $\widetilde{\mathbf{q}}$ can be organized as a linear combination of $\mathbf{s}$ independent selfstress modes:

$$
\widetilde{\mathbf{q}}=\alpha_{1} \mathbf{s}_{1}+\alpha_{2} \mathbf{s}_{2}+\cdots+\alpha_{b-r_{A}} \mathbf{s}_{b-r \sim} .
$$

Let $\alpha=\left[\begin{array}{llll}\alpha_{1} & \alpha_{2} & \cdots & \alpha_{b-r_{A}}\end{array}\right]^{T} \in \mathbf{R}^{b-r_{A}}$ be the combination coefficient vector of $\mathbf{s}$ independent self-stress modes. The combinative coefficients can theoretically take any value; however, for a statically and kinematically indeterminate tensegrity structure, one needs to judge whether the system is geometric stable or not. The study in [39] proposed the concept of product force matrix $\mathbf{T}$ as follows:

$$
\mathbf{T}=\sum_{i=1}^{s} \alpha_{i} \mathbf{G}_{i}^{T} \mathbf{M}
$$

where $\mathbf{M}$ represents the inextensional mechanisms matrix defined in equation (12) and matrix $\mathbf{G}$ is the geometric forces.

The positive definiteness of the product force matrix $\mathbf{T}$ can be determined by its eigenvalues. It is acknowledged that there is a unique distribution form of prestress for the tensegrity structure that possesses a single state of self-stress mode $(s=1)$. For structures owning multiple states of selfstress modes $(s>1)$, some mathematical optimization algorithms presented in Section 2 should be employed.

\section{Mathematical Optimization Model}

Let $p$ denote the number of positive eigenvalues of matrix $\mathbf{T}$; the objective function of stable prestress can then be set as

$$
\begin{array}{ll}
\text { minimize } & f(\alpha)=m-p \\
\text { subject to } & \alpha_{i} \in[-1,1],
\end{array}
$$

where $m$ represents the number of structural infinitesimal mechanisms defined in equation (10).

Obviously, for a given statically and kinematically indeterminate tensegrity structure, the system is geometric stable if the objective function meets the condition that $f(\alpha)=0$.

Apart from the requirement of geometric stability, stress unilateral properties of structural members need to be considered. That is to say, cables and struts must be in tension and compression, respectively. Let $u$ and $v$ be the number of tensioned cables and compressed struts, respectively. The optimization model can be adjusted into

$$
\begin{array}{ll}
\text { minimize } & f(\alpha) \\
\text { subject to } & \left\{\begin{array}{l}
\alpha_{i} \in[-1,1], \\
q_{i}>0, \quad i=1,2, \ldots u, \\
q_{i}<0, \quad i=b-v+1, \ldots, b .
\end{array}\right.
\end{array}
$$

It should be noted that strict constraint condition might result in nonsolution or nonconvergence of the optimization problem. To avoid this phenomenon, constraints can be reduced to furthest meet the condition of stress unilateral property. Let $Z$ be the number of members that coincidence with the constraint condition list in equation (16); thus,

$$
g(\alpha)=u+v-Z .
$$

Hence, one can change the optimization problem into minimizing functions $f(\alpha)$ and $g(\alpha)$ at the same time. This is a problem of multiobjective optimization. To switch it into a single objective function and, at the same time, take the priority of these two objective functions into account, two weight coefficients $\left(k_{1}, k_{2}\right)$ are introduced:

$$
h(\alpha)=k_{1} f(\alpha)+k_{2} g(\alpha) .
$$

Practically, one wishes that the obtained initial prestress can guarantee the geometric stability condition and then meet the stress unilateral condition as much as possible. In other words, the priority of function $g(\alpha)$ is expected to be lower than that of $f(\alpha)$. To reflect this relationship, the following conditions need to be satisfied for any two sets $\alpha_{1}$ and $\alpha_{2}$ :

(i) If $f\left(\alpha_{1}\right)<f\left(\alpha_{2}\right)$, for $g\left(\alpha_{1}\right)$ and $g\left(\alpha_{2}\right)$, no matter which one is larger, the relationship $h\left(\alpha_{1}\right)<h\left(\alpha_{1}\right)$ is valid. 
(ii) If $f\left(\alpha_{1}\right)=f\left(\alpha_{2}\right)$ and $g\left(\alpha_{1}\right)<g\left(\alpha_{2}\right)$, then the relationship $h\left(\alpha_{1}\right)<h\left(\alpha_{2}\right)$ is valid. That is to say, in this case, the size relation of $h\left(\alpha_{1}\right)$ and $h\left(\alpha_{2}\right)$ coincides with that of $g\left(\alpha_{1}\right)$ and $g\left(\alpha_{2}\right)$.

Hence, let $k_{1}=u+v$ and $k_{2}=1$; equation (18) can then be rewritten as

$$
h(\alpha)=(u+v) f(\alpha)+g(\alpha) .
$$

Thus, the optimization model which considered the condition of stress unilateral property can be expressed as follows:

$$
\begin{array}{ll}
\text { minimize } & h(\alpha) \\
\text { subject to } & \alpha_{i} \in[-1,1] .
\end{array}
$$

Finally, to make full use of the material, especially for the purpose of engineering construction, the initial force densities of structural members are expected to be as uniform as possible. In other words, one needs to minimize the degree of dispersion between elemental force densities of the specified groups. Accordingly, in this paper, the standard deviation function of the absolute value of the elemental force densities is employed to measure its uniformity:

$$
\operatorname{minimize} \quad l(\alpha)=\frac{\sqrt{(1 / b) \sum_{i=1}^{b}\left(\left|q_{i}\right|-(1 / b) \sum_{i=1}^{b}\left|q_{i}\right|\right)^{2}}}{(1 / b) \sum_{i=1}^{b}\left|q_{i}\right|}
$$

subject to $\alpha_{i} \in[-1,1]$.

Also, the priority of $g(\alpha)$ is expected to be higher than that of $l(\alpha)$. Fortunately, equation (17) indicates that the value of $g(\alpha)$ is a positive integer while the result calculated from equation (21) is belonging to the region from 0 to 1 . This implies that $l(\alpha)$ will be smaller than $g(\alpha)$; accordingly, the priority relationship can be satisfied automatically.

Finally, one can organize the total objective function and the total mathematical optimization model as follows:

$$
\begin{array}{ll}
T(\alpha)=(u+v) f(\alpha)+g(\alpha)+l(\alpha), \\
\text { minimize } & T(\alpha) \\
\text { subject to } & \alpha_{i} \in[-1,1] .
\end{array}
$$

In this paper, the aforementioned minimum value problem is then solved by the artificial fish swarm algorithm described in the next section.

\section{Artificial Fish Swarm Algorithm}

The AFSA, which was developed by Li et al. [32], is a population-based optimization technique inspired by natural fish swarm schooling behaviors. Due to the efficiency in solving engineering issues, AFSA has gained vast popularity in the past few decades $[35,36,40]$. As a typical swarm intelligent algorithm, each artificial fish hunts for food via its own manner, including but not limited to random, foraging behavior and swarming and following behaviors. Each artificial fish allows mutual information communications until to obtain a global optimum. Moreover, it is unnecessary to provide the gradient information during the whole optimization process. Therefore, AFSA is widely employed in searching global optimal solutions owing to the fact that it takes full advantage of the concentrated emerging mechanism of the individual intelligence.

The basic content of the AFSA is depicted as follows: suppose, in a $d$-dimensional space, there is a fish swarm composed of $N$ artificial fish. Let $X_{i}=\left(x_{i 1}, x_{i 2}, \ldots, x_{i D}\right)$ denote the current location of an artificial fish; the food consistency (the objective function) that this specified fish at location $X_{i}$ can feel is defined by $Y_{i}=f\left(X_{i}\right)$. Visual, $\delta$, and Step represent the perceiving range, the congestion factor, and the moving step, respectively. Below are the detailed definitions of the four behaviors employed in this paper [41].

(1) Random behavior

This behavior is to describe the phenomenon that, in the Visual range of a fish, it selects a position randomly and moves towards this position. It is a default behavior.

(2) Foraging behavior

Foraging is known as the basic behavior searching for food, which is on the basis of a random forage with a tendency toward food concentration. Let $X_{j}$ represent the position in the Visual range of a fish at this current. For a mathematical minimization problem, if $Y_{j}<Y_{i}$, the artificial fish will move a Step in the direction of $\left(X_{j}-X_{i}\right)$. If not, select a new state $X_{j}$ randomly and judge whether it can meet the forward condition. The random behavior is implemented if the foraging behavior is invalid after preset try-number times. Hence, the updated position $X_{i}^{*}$ can be depicted as

$X_{i}^{*}= \begin{cases}X_{i}+\frac{X_{j}-X_{i}}{d_{i j}} \cdot \text { step } \cdot \text { rand, } & \text { if } Y_{j}<Y_{i}, \\ \text { random behavior, } & \text { otherwise }\end{cases}$

where $d_{i j}=\left\|X_{i}-X_{j}\right\|$ denotes the distance between positions $X_{i}$ and $X_{j}$; rand is uniformly generated in the range of $[0,1]$.

(3) Swarming behavior

Fish are the species that is very sensitive to the external environment, and they are often assembled in several swarms to minimize possible threats. In the fish swarm, the central position $X_{c}$ of $N_{F}$ artificial fish is explored by each artificial fish $X_{i}$ in its current neighborhood $\left(d_{i j}<\right.$ Visual). Also, fish $X_{i}$ will step 


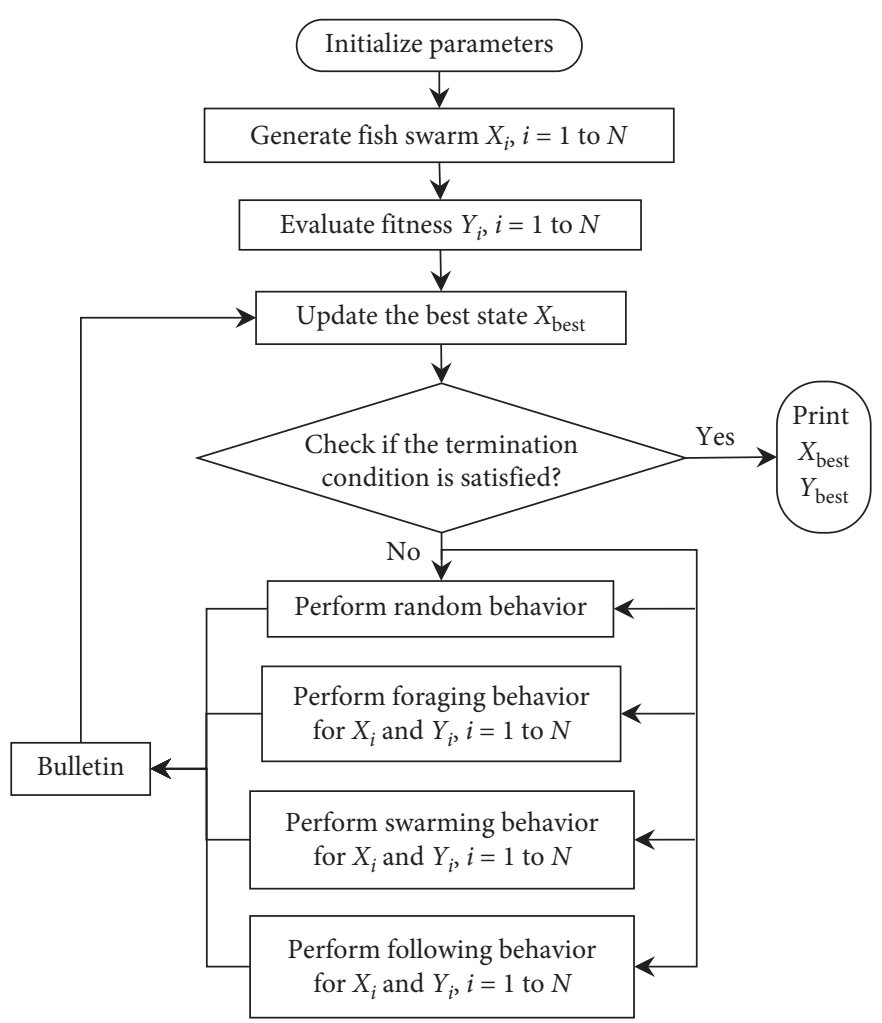

Figure 1: Flow chart of AFSA.

forward to $X_{c}$ if it satisfies the condition $Y_{c} / N_{F}<\delta \cdot Y_{i}$. Theoretically,

$$
X_{i}^{*}= \begin{cases}X_{i}+\frac{X_{c}-X_{i}}{d_{i c}} \cdot \text { step } \cdot \text { rand, } & \text { if } \frac{Y_{c}}{N_{F}}<\delta \cdot Y_{i}, \\ \text { foraging behavior, } & \text { otherwise, }\end{cases}
$$

where $\delta$ stands for the food concentration coefficient that is between 0 and 1 .

\section{(4) Following behavior}

If one fish is located at a place that has larger food concentration coefficient, other artificial fishes will follow the neighbor one to forage for food within its vision scope. Suppose $X_{\text {best }(l)}$ is the local best companion in the current neighborhood of $X_{i}$. If $\left(Y_{\text {best }(l)} / N_{F}<\delta \cdot Y_{i}\right)$, fish $X_{i}$ then attempts to step forward in the direction $\left(X_{\text {best }(l)}-X_{i}\right)$. The following behavior is described as

$$
X_{i}^{*}= \begin{cases}X_{i}+\frac{X_{\text {best }(l)}-X_{i}}{d_{i, \text { best }(l)}} \cdot \text { step } \cdot \text { rand, } & \text { if } \frac{Y_{\text {best }(l)}}{N_{F}}<\delta \cdot Y_{i}, \\ \text { foraging behavior, } & \text { otherwise. }\end{cases}
$$

The abovementioned four behaviors are implemented and compared for each artificial fish. Nevertheless, only the best behavior will be chosen to renovate the current position.
Apart from that, in a fish swarm, bulletin is utilized to record the optimum state $X_{\text {best }}$. That is to say, in each step, the state of each fish is compared to the former one, and the better state will be updated automatically in the bulletin. Figure 1 displays the flow chart to implement AFSA.

\section{Illustrative Examples}

MATLAB (version number: 2017a) platform is herein used to investigate the optimal prestress design of tensegrities. The results achieved from two illustrative examples prove that the proposed approach works remarkably well for tensegrities owing to multiple states of self-stress modes. Moreover, the accuracy and efficiency of the proposed AFSA method are also demonstrated in searching initial prestresses of the given configuration tensegrities according to practical needs.

5.1. A Planar Tensegrity Grid Beam. A planar tensegrity grid beam that has been studied by $[21,42]$ is herein used for expansion research (Figure 2(a)). Structural components displayed in the same color are thought to possess the same prestress (Figure 2(b)). According to the structural symmetry, if one divides the members into 6 groups, the system will fall into the situation in which the methods developed by $[21,42]$ are invalid and valid, respectively. Thus, such case is herein mainly discussed for comparison purpose.

The structure possesses three independent self-stress modes $(s=3)$ computed from the singular value decomposition of matrix A. Following the abovementioned 


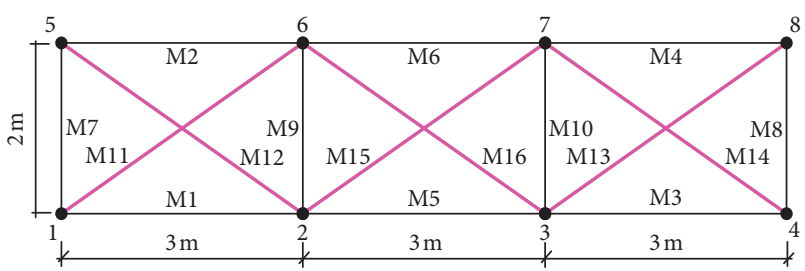

(a)

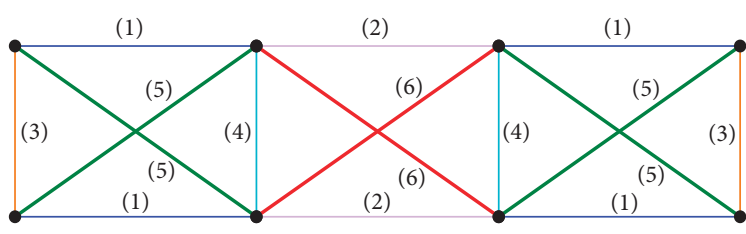

(b)

FIGURE 2: A planar tensegrity grid beam. (a) Initial configuration and (b) member group of force density assumption.

TABLE 1: Optimal prestresses of the planar tensegrity gird.

\begin{tabular}{|c|c|c|c|c|}
\hline \multirow{2}{*}{ Member group } & \multicolumn{2}{|c|}{ Feng [42] (GDA) } & \multicolumn{2}{|c|}{ Present (AFSA) } \\
\hline & Force density & $l(\alpha)$ & Force density & $l(\alpha)$ \\
\hline (1) & 1.0000 & & 1.0000 & \\
\hline (2) & 1.3997 & & 0.8893 & \\
\hline (3) & 1.0000 & & 1.0000 & \\
\hline (4) & 2.3997 & 0.3625 & 1.8893 & 0.3162 \\
\hline (5) & -1.0000 & & -1.0000 & \\
\hline (6) & -1.3997 & & -0.8893 & \\
\hline
\end{tabular}

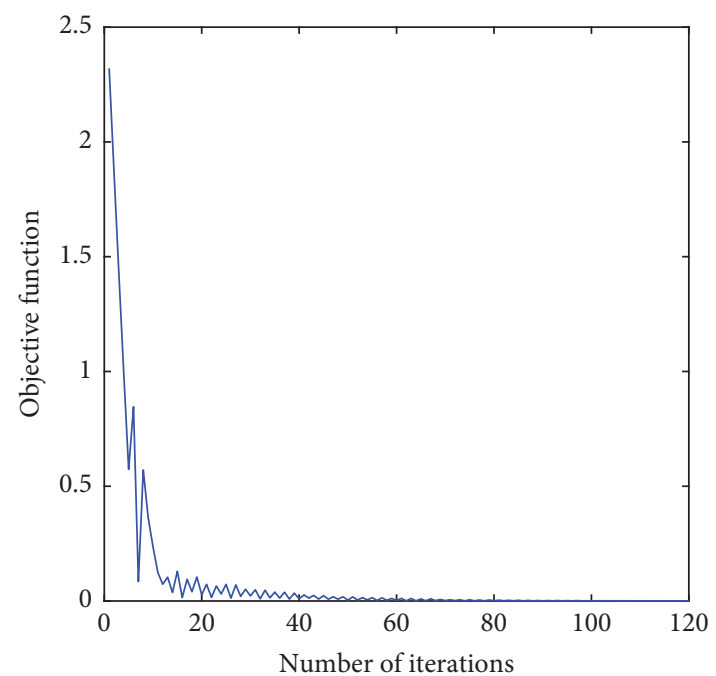

(a)

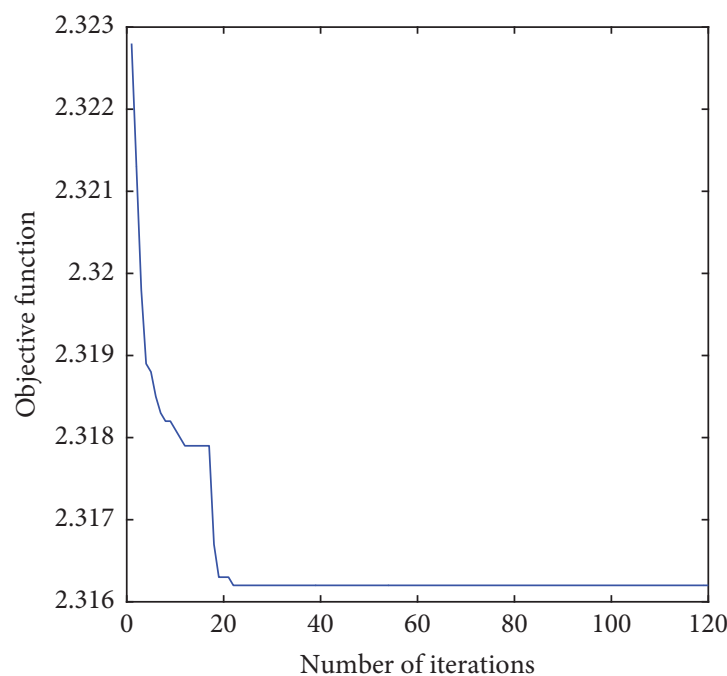

(b)

FIGURE 3: Convergence processes of the objective function for the planar tensegrity grid beam. (a) Reference [42] (GDA) and (b) present (AFSA).

mathematical model, the presented AFSA is utilized to optimize the objective function. In this example, the parameters in the AFSA are set as follows: $N=100$ (number of artificial fish), MaxGen $=50$ (maximum number of iterations), Try_number $=100$ (maximum test number of prey), Step $=0.08$ (moving step), Delta $=0.18$ (congestion factor), and Visual $=1.0$ (perception of distance) .

Table 1 depicts the obtained optimal force density of each member group and the standard deviation $l(\alpha)$ calculated from equation (21), including the gradient descent algorithm (GDA) adopted in [42] and the presented AFSA. It is easy to see that the result achieved from GDA or ASFA satisfies the unilateral condition; accordingly, the obtained force density is the feasible prestress of this tensegrity structure. It is worth noting that, however, the value of $l(\alpha)$ computed from GDA is 0.362 while, for the AFSA, it equals 0.3162. This corroborates the global searching ability of AFSA in prestress designing of tensegrity structures. The design error obtained (AFSA) is $8.7185 \times 10^{-15}$, which is within the scope from $10^{-12}$ to $10^{-16}$, confirming the accuracy of this approach. More importantly, Figure 3 gives the comparison of the convergence processes of GDA and AFSA; the results show that AFSA converges much faster than that of GDA, which demonstrates the effectiveness of the proposed AFSA method.

The eigenvalue vector achieved is [0.0000, 0.0000, 0.0000, $0.0000,0.0000,0.0338,0.1228,0.1981]^{T}$, which clearly 


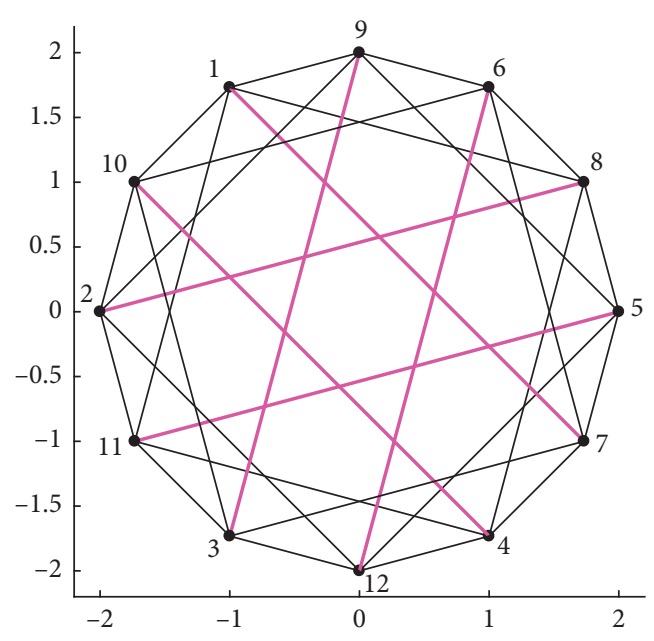

(a)

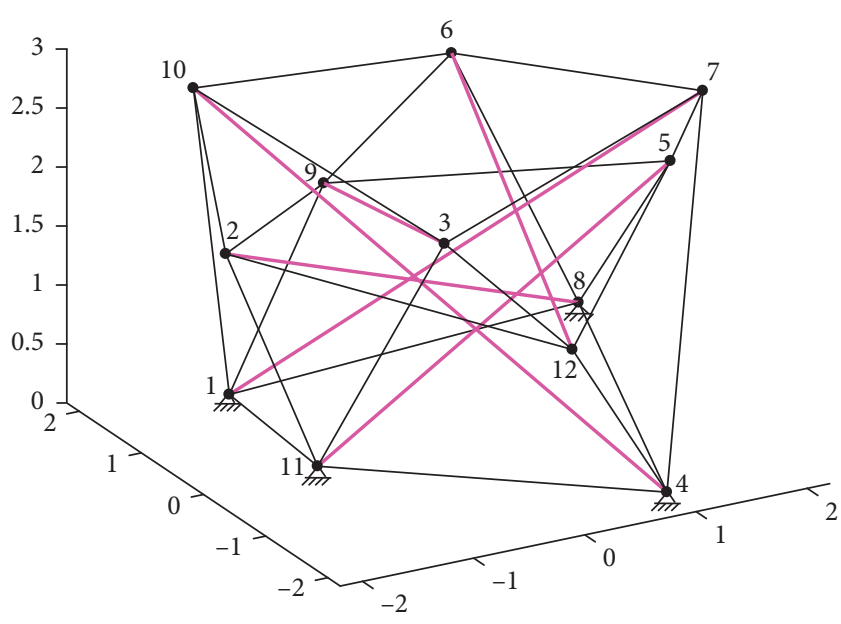

(b)

FIGURE 4: Geometry configuration of the spatial 6 struts tensegrity structure. (a) Overhead view and (b) perspective view.

TABLE 2: The feasible and uniform solutions of the initial self-stress modes.

\begin{tabular}{|c|c|c|c|c|c|c|c|c|}
\hline \multirow{2}{*}{ Element } & \multicolumn{6}{|c|}{ Independent self-stress modes } & \multirow{2}{*}{ Feasible solution } & \multirow{2}{*}{ Uniform solution } \\
\hline & 1 & 2 & 3 & 4 & 5 & 6 & & \\
\hline 1 & 0.0000 & 0.0000 & 0.0000 & 0.0000 & 0.0000 & 0.6957 & 0.2581 & 0.0933 \\
\hline 2 & 0.4591 & 0.1106 & 0.2260 & 0.0779 & -0.2576 & 0.2331 & 0.3027 & 0.0966 \\
\hline 3 & -0.2513 & -0.0454 & 0.1434 & 0.0301 & 0.2535 & -0.0318 & 0.1581 & 0.0849 \\
\hline 4 & -0.1045 & 0.1793 & -0.2076 & 0.3980 & 0.1498 & 0.0516 & 0.4915 & 0.1054 \\
\hline 5 & 0.0191 & 0.3259 & -0.2514 & 0.4052 & 0.2368 & 0.0090 & 0.4655 & 0.0696 \\
\hline 6 & 0.0773 & -0.0445 & 0.2043 & 0.0915 & 0.2481 & -0.0991 & 0.1376 & 0.0671 \\
\hline 7 & -0.2062 & 0.1181 & 0.0121 & -0.0790 & -0.3857 & -0.0430 & 0.1115 & 0.0400 \\
\hline 8 & -0.2013 & 0.5075 & 0.2225 & -0.2045 & -0.2818 & -0.0081 & 0.3089 & 0.0988 \\
\hline 9 & 0.0735 & -0.2800 & -0.0326 & 0.2796 & -0.0270 & 0.0385 & 0.1851 & 0.0699 \\
\hline 10 & -0.3498 & 0.2213 & 0.1655 & -0.0638 & 0.0864 & 0.0979 & 0.3157 & 0.1236 \\
\hline 11 & -0.2773 & -0.1574 & 0.2931 & -0.2053 & 0.0860 & 0.1891 & 0.0281 & 0.0869 \\
\hline 12 & 0.1096 & 0.0874 & 0.1530 & 0.1149 & 0.0670 & -0.2260 & 0.1786 & 0.0572 \\
\hline 13 & -0.0643 & 0.2441 & 0.0016 & 0.2857 & -0.2094 & -0.1029 & 0.5418 & 0.1446 \\
\hline 14 & -0.0849 & 0.0485 & 0.1412 & 0.0078 & -0.0914 & -0.0929 & 0.1629 & 0.0700 \\
\hline 15 & 0.0402 & -0.0234 & 0.3864 & 0.1305 & 0.2678 & -0.2061 & 0.3122 & 0.1451 \\
\hline 16 & 0.1041 & 0.0471 & 0.1342 & 0.0662 & 0.0843 & 0.3133 & 0.2763 & 0.0996 \\
\hline 17 & -0.1006 & -0.0627 & 0.1406 & 0.1389 & -0.2825 & -0.0300 & 0.3449 & 0.1374 \\
\hline 18 & -0.2019 & -0.0348 & 0.1445 & 0.0322 & 0.2161 & -0.0151 & 0.1625 & 0.0833 \\
\hline 19 & 0.1456 & 0.0494 & 0.3249 & 0.0937 & 0.0249 & 0.1647 & 0.4029 & 0.1612 \\
\hline 20 & 0.0584 & 0.1666 & 0.0092 & 0.1637 & -0.0038 & 0.1403 & 0.3448 & 0.0902 \\
\hline 21 & 0.0446 & -0.1035 & 0.1388 & 0.2548 & -0.2750 & -0.1616 & 0.3690 & 0.1358 \\
\hline 22 & -0.1683 & -0.1065 & -0.0154 & 0.1142 & -0.0499 & 0.0628 & 0.1796 & 0.0717 \\
\hline 23 & -0.3801 & -0.2597 & -0.0192 & 0.2323 & -0.1268 & 0.1409 & 0.3759 & 0.1572 \\
\hline 24 & 0.1337 & -0.3760 & 0.1666 & 0.0901 & -0.2091 & -0.0643 & 0.0131 & 0.0531 \\
\hline 25 & -0.1163 & -0.1360 & -0.1756 & -0.1736 & -0.0462 & -0.1570 & -0.4440 & -0.1446 \\
\hline 26 & 0.0332 & -0.0187 & -0.2982 & -0.0750 & -0.0846 & 0.1704 & -0.2725 & -0.1229 \\
\hline 27 & 0.1055 & -0.1664 & -0.0719 & -0.1880 & 0.1323 & 0.1035 & -0.4243 & -0.1287 \\
\hline 28 & 0.3000 & 0.1600 & 0.0515 & -0.2433 & 0.0669 & -0.1135 & -0.3664 & -0.1353 \\
\hline 29 & 0.0333 & 0.1046 & -0.0823 & -0.2034 & 0.1926 & -0.0137 & -0.3343 & -0.1249 \\
\hline 30 & 0.0152 & -0.0125 & -0.2781 & -0.0752 & -0.1289 & -0.0946 & -0.3361 & -0.1441 \\
\hline
\end{tabular}

Note: the obtained coefficient vectors to form the feasible solution and uniform solution are $(-0.3821,0.5419,0.7749,1.3248,-0.2074,0.3710)$ and $(-0.1729$, $0.0788,0.3856,0.3942,-0.0707,0.1341)$, respectively.

confirms the positive semidefinite property of matrix E, indicating the obtained tensegrity grid is definitely superstable with the ignorance of materials and prestress levels.
The structure obtained is statically indeterminate and kinematically determinate due to the fact that it has no infinitesimal mechanism with 3 rigid body motions neglected. 
5.2. A Spatial 6-Strut Tensegrity Structure. A more complicated spatial tensegrity structure, composing of 12 nodes, 6 struts, and 24 cables, is utilized for further investigation (Figure 4). Analysis of the structural equilibrium matrix A demonstrates the fact that this system possesses 6 independent self-stress modes $(s=6)$ with zero in-extensional mechanism $(m=0)$, indicating this structure is geometrical stable automatically. The AFSA is employed to optimize the objective function according to the mathematical model presented in Section 3. The parameters are set as follows: $N=100$ (number of artificial fish), MaxGen $=50$ (maximum number of iterations), Try_number $=100$ (maximum test number of prey), Step $=0.1 \quad$ (moving step), Delta $=0.523$ (congestion factor), and Visual $=2.0$ (perception of distance).

Table 2 displays the independent self-stress modes and optimized initial self-stress of the 6-strut tensegrity structure. Figure 5 shows the ladder-like convergent curve of the objective function, in which two stages are shown clearly during the optimization process. The results imply that the feasible prestress solution that only meets the unilateral condition (initial prestresses of the first 24 cables are positive, while they are negative for the last 6 struts) is obtained in the first stage. The uniform prestress solution is acquired subsequently after a few iterations. Table 2 lists the feasible and uniform solutions of the initial prestress modes; as can be seen, the distribution of prestresses is highly dispersive before the optimization of uniformity is implemented; for example, the ratio of the maximum prestress (0.5418) and minimum prestress $(0.0131)$ is up to 41.3588 while it decreases to 4.0300 after the optimization of uniformity is carried out, which strongly verifies the validity of the presented AFSA approach. For this instance, the design error achieved is $1.0450 \times 10^{-15}$, which confirms the accuracy of the proposed approach. Additionally, it can be found that the objective function achieved from AFSA is close to the minimum value 0.32605 after about 30 iterations (Figure 5), confirming the efficacy of this algorithm.

\section{Conclusions}

An effective approach is presented for optimal initial selfstress design of tensegrity structures based on AFSA algorithm. First of all, the process is executed by dealing with a linear homogeneous system concerning the self-equilibrium system. The issue is subsequently performed as a minimum problem by regulating the value of an objective function where the unilateral condition and the stress uniformity condition are fully considered. The AFSA is adopted to search for the global minimum solution that guarantees all the above conditions. Two illustrative examples were fully investigated in demonstrating the accuracy and efficiency of the proposed AFSA method. The results confirm that AFSA has advantages in overcoming the local minima and has rapid convergence during the process. It should be noted that although AFSA adopted in this work produces good quality solutions, the initial design points utilized in the illustrative examples are determined by a number of tentative calculations. During this procedure, the initial design

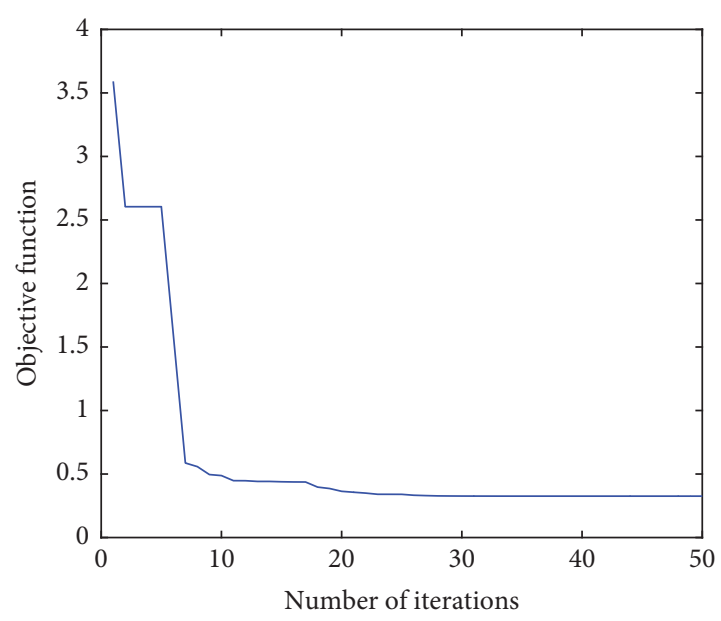

Figure 5: Convergence process of the objective function for the spatial 6-strut tensegrity structure.

points that cause the program failure in obtaining results or results that do not converge are eliminated. Computational methods that could mitigate the selection numbers of initial design points might be the future direction. Furthermore, some advanced optimization approaches, such as the Monte Carlo optimization and the genetic algorithm, might also be integrated in topology design of more complicated tensegrities.

\section{Data Availability}

All data, models, or codes generated or used during the study are available from the corresponding author on request.

\section{Conflicts of Interest}

The authors declare that they have no conflicts of interest.

\section{Acknowledgments}

This work was supported by the National Natural Science Foundation of China (Grant no. 51908356), the Foundation of China Postdoctoral Science (Grant no. 2019M662056), the Natural Science Foundation of Zhejiang Province (Grant no. LQ19E080013), and the International Scientific and Technological Cooperation Projects of Shaoxing University (Grant no. 2019LGGH1005).

\section{References}

[1] A. G. Tibert and S. Pellegrino, "Deployable tensegrity reflectors for small satellites," Journal of Spacecraft and Rockets, vol. 39, no. 5, pp. 701-709, 2002.

[2] D. E. Ingber, "Tensegrity I. Cell structure and hierarchical systems biology," Journal of Cell Science, vol. 116, no. 7, pp. 1157-1173, 2003.

[3] D. Stamenović, "Effects of cytoskeletal prestress on cell rheological behavior," Acta Biomaterialia, vol. 1, no. 3, pp. 255-262, 2005. 
[4] S. S. Simmel, P. C. Nickels, and T. Liedl, "Wireframe and tensegrity DNA nanostructures," Accounts of Chemical Research, vol. 47, no. 6, pp. 1691-1699, 2014.

[5] R. Connelly and A. Back, "Mathematics and tensegrity," American Scientist, vol. 86, no. 2, pp. 142-151, 1998.

[6] F. Fu, "Structural behavior and design methods of tensegrity domes," Journal of Constructional Steel Research, vol. 61, no. 1 , pp. 23-35, 2005.

[7] C. Paul, F. J. Valero-Cuevas, and H. Lipson, "Design and control of tensegrity robots for locomotion," IEEE Transactions on Robotics, vol. 22, no. 5, pp. 944-957, 2006.

[8] A. G. Rovira and J. M. M. Tur, "Control and simulation of a tensegrity-based mobile robot," Robotics and Autonomous Systems, vol. 57, no. 5, pp. 526-535, 2009.

[9] K. Caluwaerts, J. Despraz, A. Işçen et al., "Design and control of compliant tensegrity robots through simulation and hardware validation," Journal of the Royal Society Interface, vol. 11, no. 98, 2014.

[10] R. Motro, S. Najari, and P. Jouanna, "Static and dynamic analysis of tensegrity systems," in Proceedings of the International Symposium On Shell and Spatial Structures: Computational Aspects, pp. 170-179, Springer, Leuven, Belgium, March 1987, Lecture Notes in Engineering.

[11] N. Veuve, A. C. Sychterz, and I. F. C. Smith, "Adaptive control of a deployable tensegrity structure," Engineering Structures, vol. 152, pp. 14-23, 2017.

[12] X.-F. Yuan, S. Ma, and S.-H. Jiang, "Form-finding of tensegrity structures based on the Levenberg-Marquardt method," Computers \& Structures, vol. 192, pp. 171-180, 2017.

[13] X. Feng, Y. Ou, and M. S. Miah, "Energy-based comparative analysis of optimal active control schemes for clustered tensegrity structures," Structural Control \& Health Monitoring, vol. 25, no. 10, p. e2215, 2018.

[14] Y. Chen, J. Yan, J. Feng, and P. Sareh, "A hybrid symmetryPSO approach to finding the self-equilibrium configurations of prestressable pin-jointed assemblies," Acta Mechanica, vol. 231, no. 4, pp. 1485-1501, 2020.

[15] J. H. Lee and M. L. Shofner, "Tensegrity-inspired polymer nanocomposites," Polymer, vol. 111, pp. 9-19, 2017.

[16] H.-J. Schek, "The force density method for form finding and computation of general networks," Computer Methods in Applied Mechanics and Engineering, vol. 3, no. 1, pp. 115-134, 1974.

[17] M. Pagitz and J. M. M. Jur, "Tensegrity frameworks: static analysis review," Mechanism and Machine Theory, vol. 43, no. 7, pp. 859-881, 2008.

[18] J. Y. Zhang and M. Ohsaki, "Adaptive force density method for form-finding problem of tensegrity structures," International Journal of Solids \& Structures, vol. 43, no. 18-19, pp. 5658-5673, 2006.

[19] G. G. Estrada, H. J. Bungartz, and C. Mohrdieck, "Numerical form-finding of tensegrity structures," International Journal of Solids and Structures, vol. 43, no. 22-23, pp. 6855-6868, 2006.

[20] H. C. Tran and J. Lee, "Advanced form-finding of tensegrity structures," Computers \& Structures, vol. 88, no. 3-4, pp. 237-246, 2010.

[21] H. C. Tran and J. Lee, "Form-finding of tensegrity structures using double singular value decomposition," Engineering with Computers, vol. 29, no. 1, pp. 1-16, 2013.

[22] H. C. Tran and J. Lee, "Initial self-stress design of tensegrity grid structures," Computers \& Structures, vol. 88, no. 9-10, pp. 558-566, 2010.

[23] H. C. Tran and J. Lee, "Determination of a unique configuration of free-form tensegrity structures," Acta Mechanica, vol. 220, no. 1-4, pp. 331-348, 2011.
[24] J. Y. Zhang and M. Ohsaki, "Force identification of prestressed pin-jointed structures," Computers \& Structures, vol. 89, no. 23-24, pp. 2361-2368, 2011.

[25] K. Koohestani and S. D. Guest, "A new approach to the analytical and numerical form-finding of tensegrity structures," International Journal of Solids and Structures, vol. 50, no. 19, pp. 2995-3007, 2013.

[26] S. Lee and J. Lee, "Form-finding of tensegrity structures with arbitrary strut and cable members," International Journal of Mechanical Sciences, vol. 85, no. 8, pp. 55-62, 2014.

[27] M. Quagliaroli, G. Malerba, A. Albertin, and N. Pollini, "The role of prestress and its optimization in cable domes design," Computers \& Structures, vol. 161, pp. 17-30, 2015.

[28] L.-Y. Zhang, Y. Li, Y.-P. Cao, and X.-Q. Feng, "Stiffness matrix based form-finding method of tensegrity structures," Engineering Structures, vol. 58, no. 7, pp. 36-48, 2014.

[29] S. Lee, J. Lee, and J.-W. Kang, "Results of generalized equilibrium path from form-finding of tensegrity structure," International Journal of Steel Structures, vol. 17, no. 3, pp. 1225-1231, 2017.

[30] K. Koohestani, "On the analytical form-finding of tensegrities," Composite Structures, vol. 166, pp. 114-119, 2017.

[31] X. Feng, "An investigation on optimal initial self-stress design of tensegrity grid structures," International Journal of Steel Structures, vol. 18, no. 3, pp. 960-975, 2018.

[32] X. L. Li, Z. J. Shao, and J. X. Qian, "An optimizing method based on autonomous animate: fish swarm algorithm," Systems Engineering-Theory \& Practice, vol. 22, pp. 32-38, 2002.

[33] M. Ma, J. H. Liang, L. Sun, and M. Wang, "SAR image segmentation based on SWT and improved AFSA," in Proceedings of the Third International Symposium on Intelligent Information Technology and Security Informatics, pp. 146-149, IEEE Computer Society Washington, Jinggangshan, China, April 2010.

[34] W. J. Tian and J. C. Liu, "A novel approach to fuzzy logic controller design," in Proceedings of the Second International Conference on Computer Modeling and Simulation, pp. 112115, IEEE Computer Society Washington, Sanya, China, January 2010

[35] R. Huang, H. Tawfik, A. Nagar, and G. Abbas, "A novel hybrid Qos multicast routing based on clonal selection and artificial fish swarm algorithm," in Proceedings of the Second International Conference on Developments in eSystems Engineering, pp. 47-52, IEEE Computer Society Washington, Abu Dhabi, UAE, December 2009.

[36] Y. M. Cheng, L. Liang, S. C. Chi, and W. B. Wei, "Determination of the critical slip surface using artificial fish swarms algorithm," Journal of Geotechnical and Geoenvironmental Engineering, vol. 134, no. 2, pp. 244-251, 2008.

[37] X. Feng and S. Guo, "A novel method of determining the sole configuration of tensegrity structures," Mechanics Research Communications, vol. 69, pp. 66-78, 2015.

[38] S. Pellegrino, "Structural computations with the singular value decomposition of the equilibrium matrix," International Journal of Solids and Structures, vol. 30, no. 21, pp. 3025-3035, 1993.

[39] S. Pellegrino and C. R. Calladine, "Matrix analysis of statically and kinematically indeterminate frameworks," International Journal of Solids and Structures, vol. 22, no. 4, pp. 409-428, 1986.

[40] X. L. Li, F. Lu, and G. H. Tian, "Applications of artificial fish school algorithm in combinatorial optimization problem," Journal of Shandong University, vol. 34, no. 5, pp. 64-67, 2004. 
[41] B. Xing and W. J. Gao, Innovative Computational Intelligence: A Rough Guide to 134 Clever Algorithms, Springer International Publishing, Cham, Switzerland, 2014.

[42] X. Feng, "The optimal initial self-stress design for tensegrity grid structures," Computers \& Structures, vol. 193, pp. 21-30, 2017. 